Pour une anthropologie des pratiques sportives. Propriétés formelles et rapport au corps dans le rugby à XV

\title{
Sébastien Darbon
}

\section{(2) OpenEdition}

Journals

Édition électronique

URL : https://journals.openedition.org/tc/153

DOI : $10.4000 /$ tc. 153

ISSN : 1952-420X

Éditeur

Éditions de l'EHESS

Édition imprimée

Date de publication : 1 juin 2002

ISSN : 0248-6016

Référence électronique

Sébastien Darbon, «Pour une anthropologie des pratiques sportives. Propriétés formelles et rapport au corps dans le rugby à XV », Techniques \& Culture [En ligne], 39| 2002, mis en ligne le 29 avril 2005, consulté le 29 septembre 2022. URL : http://journals.openedition.org/tc/153 ; DOl : https://doi.org/ $10.4000 /$ tc. 153

Ce document a été généré automatiquement le 29 septembre 2022.

Tous droits réservés 


\title{
Pour une anthropologie des pratiques sportives. Propriétés formelles et rapport au corps dans le rugby à XV
}

\author{
Sébastien Darbon
}

1 Si l'on en juge par la quantité des publications qui leur sont consacrées, il semble que les pratiques sportives sont encore rarement considérées, tout au moins en France, comme un objet stimulant pour la réflexion anthropologique. Les raisons d'une telle méfiance sont sans doute à trouver dans le décentrement assez radical qu'impose, au sein des exigences de l'anthropologie « canonique », la prise en compte de phénomènes culturels issus des sociétés modernes contemporaines. Lorsque, cependant, des phénomènes qui ne sont pas sans rappeler les pratiques sportives sont observés au sein de sociétés traditionnelles, leur évocation retrouve une certaine forme de légitimité. Ainsi, Claude Lévi-Strauss, analysant le rituel des jeux de balle entre Tokanagi et Kickoagi et le fait que l'issue de la partie est déterminée non par les forces en présence mais par la nécessité que triomphe toujours le « camp des morts », peut affirmer :

« La structure formelle de ce qui, au premier abord, pourrait apparaître comme une compé-tition sportive, est en tous points similaire à celle d'un pur rituel, tel que le Mitawit ou Midewiwin des mêmes sociétés algonkines, où les néophytes se font symboliquement tuer par les morts dont les initiés jouent le rôle, afin d'obtenir un supplément de vie réelle au prix d'une mort simulée. » (Lévi-Strauss 1962 : 46).

2 En d'autres termes, ce qui justifie ici l'intérêt que l'on peut porter à des pratiques évoquant une activité sportive, ce n'est pas tant le contenu même de ces pratiques, mais le fait qu'elles prennent place au sein d'une société traditionnelle.

Par ailleurs, l'extension planétaire de ces pratiques (malgré des inégalités de diffusion géographique qui ne sont pas sans poser, comme nous le verrons plus loin, de très intéressants problèmes culturels), jointe à la mobilisation de foules considérables qu'elles entrainent souvent, semble produire un effet d'échelle a priori peu compatible avec la tradition de l'enquête de terrain et de l'observation participante au sein de 
petites communautés qui sont censées être caractérisées par une grande homogénéité culturelle.

4 Certes, des exceptions notables ont récemment montré qu'il n’y avait là rien d'essentiellement fatal. Pour s'en tenir aux travaux français, Christian Bromberger $(1995,1998)$ a produit une analyse ethnologique très riche des supporteurs de football qui éclaire les raisons de l'engouement de certains de nos contemporains pour ce type de spectacle sportif et trace les contours d'une véritable culture. À propos d'un autre sport, le rugby, Anne Saouter met en scène les jeux du masculin et du féminin au sein d'une communauté pour laquelle la virilité est une valeur de référence fondamentale, contribuant ainsi à alimenter, à partir de "terrains » sportifs, un champ de réflexions -le genre- curieusement sous-investi dans les recherches anthropologiques françaises ${ }^{1}$ (Saouter 2000).

5 Par rapport à ces avancées manifestes dans la perspective de l'élaboration d'une réflexion anthropologique, notre propos dans cet article est à la fois plus global et plus ambitieux. Plus global en ce qu'il prend pour objet l'ensemble des "pratiquants » (joueurs, dirigeants, supporteurs...) et non seulement les spectateurs dans les tribunes, mais aussi l'ensemble des sports (ce qui n'exclut pas, bien au contraire, des différenciations fines au sein de ces derniers); plus ambitieux dans la mesure où il s'agit de mettre en évidence, non la seule existence de cultures sportives spécifiques (aussi nombreuses qu'il y a de sports différents), mais encore les modalités selon lesquelles se structure une culture sportive donnée - notamment à travers l'examen approfondi des propriétés formelles de la pratique.

6 Cette réflexion a notamment été nourrie par divers travaux ethnographiques réalisés dans une perspective comparative. Une première investigation nous avait conduit à Saint-Vincent-de-Tyrosse, petit bourg des Landes situé à mi-chemin entre Dax et Bayonne, où s'exprime en toute légitimité l'essence même de cette culture traditionnelle du rugby (Darbon 1995a). Le premier élément de comparaison avait ensuite été obtenu en faisant varier de façon radicale le contexte dans lequel s'insère la pratique du rugby : en choisissant d'étudier un club de Marseille, il s'agissait de faire jouer l'opposition entre "rugby des villes» et «rugby des villages", mais aussi d'évaluer dans quelle mesure la culture traditionnelle du rugby peut parvenir à s'implanter dans un environnement hostile, entièrement voué au football (Darbon 1997a, 1999).

7 De cette comparaison découlait assez naturellement la question de la dimension «universelle» (ou au contraire contingente) de certains éléments de la culture rugbystique: dans la transplantation d'un contexte à un autre, quels étaient les éléments qui restaient inchangés, quels étaient ceux qui disparaissaient ou subissaient des variations importantes? L'hypothèse qui se dégageait alors était celle d'une importance fondamentale des contraintes formelles (et au sein de celles-ci, des règles $d u$ jeu) dans la structuration d'une culture sportive spécifique.

8 Il restait à faire jouer un deuxième élément de comparaison, non plus entre contextes différents, mais entre pratiques sportives différentes, c'est-à-dire caractérisées par des règles du jeu différentes. Dans cette perspective, il a paru pertinent d'opposer deux sports collectifs à la fois suffisamment proches l'un de l'autre pour que la comparaison soit valable et suffisamment distincts par leurs règles pour que l'hypothèse de l'influence de celles-ci puisse être testée : le rugby à XV (dans ses diverses inscriptions 
contextuelles) et le rugby à XIII (à partir d'une analyse monographique réalisée dans le club de Carpentras).

9 Les hypothèses de travail présentées ci-après, sous la forme d'un modèle théorique, s'inspirent abondamment des résultats de ces monographies, manifestant ainsi la préférence pour une approche plus inductive qu'hypothético-déductive. Mais la nécessité de confronter à leur tour ces hypothèses à de nombreuses vérifications empiriques trace les contours d'un vaste chantier dont nous espérons qu'il contribuera à donner à la réflexion anthropologique sur les pratiques sportives la légitimité qui semble lui faire encore défaut.

Fondements du « modèle »

10 L'emploi du terme "modèle », plus familier dans une discipline comme la science économique que dans celles qui, comme l'anthropologie, valorisent presque exclusivement l'approche qualitative, peut surprendre. On entend en effet par là une représentation simplifiée de la réalité à partir de la définition d'un nombre relativement restreint de variables jugées pertinentes et des liens que ces variables entretiennent les unes avec les autres. Or, si la définition de variables pertinentes ne pose pas de problèmes, celle de leurs liens réciproques reçoit, dans le cas qui nous intéresse, une réponse spécifique qui n'est ni mathématique, ni statistique, mais purement qualitative : davantage rapports d'évidence que de corrélation, d'affinité que de causalité, les liens mis en scène ne tiennent que par la rigueur de l'argumentation proposée et sa capacité à résister à des argumentations concurrentes. En outre, il peut paraître paradoxal de valoriser un processus de simplification du réel dans une discipline qui se nourrit au contraire de la complexité de celui-ci. Mais on pourra d'abord rappeler que l'anthropologie est en permanence (et, pourrait-on dire, par définition) traversée de ces mouvements contraires qui, tantôt insistent sur l'infinie richesse des diversités culturelles, tantôt essaient de mettre en évidence des régularités, des ressemblances, voire des invariances. Ensuite, on soulignera qu'une telle simplification n'est acceptable et pertinente que si les hypothèses qui la fondent sont elles-mêmes acceptables et pertinentes, c'est-à-dire si elles sont informées par un travail ethnographique approfondi. Enfin, l'insistance sur cet ensemble particulier d'hypothèses nous paraît indispensable, au sens où le fait de les négliger conduirait à ne rien comprendre aux cultures sportives; mais une telle position n'est en aucune façon à visée hégémonique, en ce sens qu'elle n'implique pas le rejet d'analyses complémentaires : par exemple, il est bien clair que l'assimilation par les pratiquants des catégories culturelles spécifiques à leur sport passe par une certaine forme d'enseignement ou d'apprentissage, par la reproduction de schèmes observés chez les autres pratiquants, etc. ${ }^{2}$ Il ne s'agit donc pas de nier le problème de la transmission du capital culturel et des modalités de cette transmission, mais de signaler que cette assimilation est en grande partie le produit des propriétés formelles spécifiques des pratiques considérées.

11 Dans sa version la plus simple, le modèle proposé ici énonce qu'il existe autant de cultures sportives que de pratiques sportives distinctes, et que ces cultures sont structurées pour l'essentiel par les propriétés formelles des pratiques correspondantes. Nous verrons par la suite comment il peut être raffiné et complexifié.

Des cultures sportives?

12 Nous définissons une culture sportive comme une combinaison spécifique de pratiques, de comportements, de rapports au corps et de systèmes de valeurs caractéristiques du 
groupe des pratiquants d'un sport donné3. Dans cette mesure, plutôt que de s'intéresser à ce que serait une culture sportive englobante, dont les traits seraient communs à toutes les pratiques, il convient de distinguer autant de cultures sportives qu'il y a de pratiques différenciées.

Mais pour autant, les différentes cultures sportives ne constituent pas des ensembles disjoints. Pour que deux sports fassent référence à des cultures distinctes, il n'est pas nécessaire que tous les éléments des deux combinaisons considérées soient différents, ou dans un rapport d'opposition ${ }^{4}$. Certains de ces éléments peuvent être identiques : pour reprendre l'exemple cité en note 4 , on pourra retrouver des comportements marqués par l'individualisme, à la fois en football et en rugby à XIII; d'autres éléments peuvent varier, comme le rapport au corps ou les comportements d'excès, du fait que le rugby à XIII est un sport de combat collectif, ce qui n'est pas le cas du football. Pour distinguer deux cultures sportives, il faut donc que l'on ait pu repérer, au sein de la combinaison des éléments pertinents, au moins un de ces éléments qui diffère.

Par ailleurs, du fait notamment de la relative plasticité des modèles culturels, ou si l'on préfère des individus qui s'y conforment, un pratiquant ou un groupe de pratiquants peuvent participer de diverses cultures sportives : un sportif pourra avoir pratiqué avec un égal bonheur le rugby et le basket-ball (ce qui n'est pas rare), mais dans le cadre de sa pratique du rugby, il se conformera aux exigences de la culture rugbystique (faute de quoi il serait rejeté par le groupe), alors que dans le cadre de sa pratique du basket-ball, il se comportera plutôt en joueur de basket-ball. Une telle labilité n'a rien d'exceptionnel : c'est par exemple ce à quoi nous nous plions quotidiennement lorsque nous passons (avec plus ou moins de facilités) des codes culturels qui s'imposent à nous dans notre milieu professionnel à ceux en vigueur dans l'intimité de notre foyer.

Or, ces cultures sportives ne tombent pas du ciel : nombreux sont les historiens et les sociologues des pratiques sportives qui ont, notamment en langue anglaise, montré à quel point ces dernières constituaient un phénomène contingent, historiquement déterminé par le mécanisme complexe des forces sociales, économiques, politiques qui sont à l'œuvre dans un pays donné, à un moment donné. Il est clair, par exemple, que la différenciation progressive, dans l'Angleterre du XIXe siècle, entre le football-rugby et le football-association s'est produite au nom de deux logiques portées par des groupes sociaux ayant des objectifs distincts en matière d'éducation par le sport. Et au sein même du football-rugby, on peut mettre en évidence les conditions économiques et sociologiques de la naissance du code treiziste en Angleterre à la fin du siècle dernier. Dans une étude riche et pertinente, E. Dunning et K. Sheard (1976) ont ainsi montré que, dans la rupture entre Rugby League et Rugby Union, se manifeste un antagonisme entre classes sociales qui double une opposition régionale. Alors que dans le Nord coexistent des clubs huppés, recrutant dans les public schools, et des clubs d'ouvriers et de mineurs, dans le Sud, relativement peu industrialisé, ce sont encore les clubs « aristocratiques » qui tiennent le haut du pavé. Il est naturel que la revendication par les joueurs du paiement du "manque à gagner " pour cause d'entraînement ou de blessure (amorce d'une "professionnalisation» du sport) soit venue de ces clubs populaires. Par ailleurs, l'importance des foules qui se passionnent pour le footballrugby dans certains comtés du Nord (notamment le Yorkshire et, dans une moindre mesure, le Lancashire), fournit la base économique permettant de s'acheminer vers des entrées payantes, et donc de rémunérer les joueurs. L'essentiel, pour notre pro-pos, c'est que dans un premier temps, cette monétisation du jeu introduit un certain 
nombre de contraintes qui conduisent à modifier les règles du jeu du football-rugby. Ces modifications (création d'espaces supplémentaires en diminuant le nombre de joueurs, aménagement de phases de jeu jugées trop statiques comme la mêlée fermée, où ne s'exerce plus de véritable poussée, adoption du «tenu »...) ont toutes le même objectif : rendre le jeu plus spectaculaire, plus attrayant, et donc drainer vers les stades des spectateurs payants de plus en plus nombreux. Ainsi naît une nouvelle pratique sportive, le rugby à XIII, dont les propriétés formelles ne sont plus exactement les mêmes que celles du rugby à XV.

16 L'intérêt de ce genre d'analyses est évident, notamment en ce qu'il met l'accent sur la dimension étroitement contingente de la création et de l'évolution des règles du jeu dans le domaine sportif. Il nous semble cependant qu'elles pourraient être prolongées de façon fructueuse en prenant en compte l'extraordinaire pouvoir qu'ont les propriétés formelles d'un sport donné de façonner, par elles-mêmes, des comportements et des pratiques, de les renforcer et de les constituer en systèmes de référence. Elles ne sont plus seulement conséquences d'un ensemble complexe de facteurs historiques (leur dimension en quelque sorte "passive»), mais sont alors considérées comme un formidable moteur d'élaboration et de consolidation d'une culture sportive. C'est cette dimension de structuration de la culture par l'intermédiaire des propriétés formelles que nous souhaitons mettre en évidence tout au long de ce travail, en prenant l'exemple du rugby 5 .

Des propriétés formelles de la pratique

Dans les développements qui précèdent, c'est surtout le rugby qui a été analysé; comme tous les sports individuels ou collectifs de balle ou de ballon, c'est aussi un jeu et à ce titre, il est défini, au sens le plus fort du terme, par des règles du jeu. Or, quelle que soit la difficulté que l'on éprouve à définir rigoureusement ce qu'est un sport, il est indéniable que l'on assiste depuis environ une vingtaine d'années à l'émergence, à côté des disciplines «classiques » (sports individuels et collectifs de balle, athlétisme...), de sports intitulés "fun" ou «de glisse » qui, bien que formant une nébuleuse dont les contours sont d'autant plus difficiles à cerner qu'elle est elle-même en constante et rapide évolution, sont soumis à des contraintes formelles tout autres. Ainsi, alors que les pratiquants de surf, les adeptes du parapente ou les passionnés d'escalade sont avant tout confrontés à la nature (plus ou moins) sauvage (la vague, les flux d'air, la paroi rocheuse), dans des configurations où les dispositifs compétitifs et réglementaires sont le plus souvent très marginaux ${ }^{6}$, les footballeurs, basketteurs et autres lanceurs de marteau évoluent dans un milieu beaucoup plus protégé et standardisé (le stade), et sont soumis à des contraintes au sein desquelles ce sont les règles du jeu qui dominent. Ainsi s'effectue une différenciation à deux étages. D'une part, il faut distinguer les propriétés formelles qui découlent de la contrainte que nous appellerons " naturelle » (notamment les sports dits "fun» ou "de glisse») de celles qui découlent de la contrainte que nous appellerons "réglementaire" (où les sports individuels ou collectifs de balle ou de ballon se taillent la part du lion). D'autre part, au sein de chacune de ces deux catégories, des distinctions doivent encore être faites : de même que dans le cadre des contraintes naturelles, les propriétés formelles du surf, du snowboard ou du parapente ne sont pas réductibles les unes aux autres, dans le cadre des contraintes réglementaires, les propriétés formelles du football, du rugby ou du basket-ball ne sont pas les mêmes. 

$\mathrm{XV}$, sera envisagée la mise en évidence du lien entre une contrainte de type réglementaire et la culture sportive correspondante.

À la recherche de régularités de cultures qui sont davantage inscrites dans des territoires ou liées à l'ethnicité (ce qui va souvent de pair), qu'elles ont une dimension transversale, précisément dépendante de la nécessaire universalité des règles du jeu. Bien que moins répandu que le football, le rugby est malgré tout joué dans de nombreux pays; si l'on veut que ces pays puissent se rencontrer dans le cadre de compétitions (comme c'est le cas, par exemple, avec la Coupe du Monde), il faut bien que les mêmes règles s'appliquent partout. Nous devons alors déduire logiquement des propositions précédentes qu'un certain nombre de dimensions fondamentales de la culture du rugby doivent se retrouver à Auckland comme à Bath, à Trévise comme à Toulouse. Autrement dit, on sera amené à identifier ce que nous appellerons des invariants ou des universaux ${ }^{7}$ de la culture rugbystique, quel que soit le contexte culturel local de réception. Nous verrons par la suite que la souplesse et la perméabilité des cultures sportives (comme celles de toutes formes de cultures), d'une part les soumettent aux influences du temps qui passe, et d'autre part donnent nécessairement lieu à des réinterprétations de ces configurations culturelles en fonction des contextes au sein desquels elles sont immergées : on assiste alors à un jeu dialectique complexe entre la dimension universelle et la dimension contextuelle des cultures sportives ${ }^{8}$. Pour l'instant, focalisons notre attention sur les caractéristiques de la culture du rugby, que l'on peut considérer comme indépendantes de cette nécessaire contextualisation.

Le rôle central du corps

yse de l'évolution des règles du rugby le montre bien: en l'espace d'un siècle et demi, un sport s'est constitué dans ses traits spécifiques à la suite de multiples décisions qui répondent à une certaine logique (Conquet 1994). Cette logique, dont on peut après coup analyser la cohérence, n'est partagée que par un tout petit nombre de sports collectifs de ballon -en réalité, le rugby à XIII et le football américain. Pour le mettre en évidence, il convient de faire appel au critère du type d'opposition par l'intermédiaire duquel on se saisit du ballon :

- dans la plupart des sports collectifs (football, basket-ball, handball, hockey sur gazon et sur glace, water-polo, squash, pelote basque... $\left.{ }^{10}\right)$, les adversaires s'opposent au ballon ou à la balle;

- dans les trois autres sports (rugby à XV et à XIII, football américain), on n'obtient le ballon qu'en s'opposant au corps de l'adversaire.

21 Cette distinction est tout à fait fondamentale, en ce sens qu'elle permet d'opposer, traditionnellement, les sports de «contact» et les sports de «combat». Une telle terminologie mérite cependant quelque attention. Forgée par les informateurs euxmêmes -lesquels ne sont d'ailleurs pas toujours d'accord entre eux ${ }^{11}$ - elle nécessite en effet reformulation et dépassement pour fonder une analyse ethnologique sérieuse. En rugby, sur $80 \mathrm{mn}$ de jeu (deux mi-temps de $40 \mathrm{mn}$ chacune), on compte environ 27 $m n$ de jeu réel ${ }^{12}$. Dans un match de haut niveau, les phases de jeu groupé (mêlées, touches et regroupements) interviennent en moyenne 130 fois au sein de ce jeu réel; il faut y ajouter les phases de charge et de plaquage (une centaine dans chaque cas). Fréquence impressionnante de phases d'affrontement physique dur, la cible étant le corps de l'adversaire et non le ballon. En d'autres termes, c'est parce que le corps de 
l'adversaire aura été défait que l'on pourra jouer le ballon et marquer. Pour cette raison, il nous paraît préférable de substituer la notion de combat à celle de contact.

Un auteur comme P. Parlebas (1986), dont les travaux de sociologie du sport insistent avec beaucoup de rigueur et de pertinence sur le rôle central du corps, est cependant conduit par sa logique classificatoire à ne pas distinguer fondamentalement le rugby du football (regroupés dans la catégorie des sports de contact), et d'autre part à mettre dans le même sac (celui des sports de combat) des pratiques aussi différentes, voire structurellement opposées, que la lutte gréco-romaine et le fleuret. Il est clair, pour qui n'a pas une vue trop abstraite de la réalité des pratiques sportives, que la lutte grécoromaine, dont on ne contestera pas qu'elle est un sport de combat, est beaucoup plus proche du rugby (notamment dans les modalités que prennent les affrontements en mêlée ou dans certains regroupements) qu'elle ne l'est des diverses formes d'escrime. L'erreur d'analyse tient ici à une illusion tenace, qui fait de l'objectif de chaque sport (marquer un but, porter la balle à tel endroit, toucher l'adversaire...) le principe fondamental du classement, alors que ce principe doit être relié aux modalités par lesquelles on parvient à cet objectif : interdire ou autoriser de prendre la balle à la main, de percuter l'adversaire, etc. Ce sont ces caractéristiques concrètes qui sont structurantes de morphologies, de comportements et de représentations, et non les caractéristiques abstraites liées à l'objectif. Comment s'effectue une telle structuration?

Une certaine conception du groupe

Les deux rugbys et le football américain, on l'a vu, ont pour caractéristique fondamentale commune d'être des sports d'affrontement à l'homme et non au ballon. La forme la plus spectaculaire que peut prendre cette opposition est certainement le plaquage.

25 Le vocabulaire est ici très explicite: en plaquant l'adversaire, on cherche à le "châtier ", à le «laminer ", à le "désosser "; le «tampon", le «bouchon" ou le «caramel » n'ont pas seulement pour but d'arrêter un joueur porteur du ballon, ils doivent aussi l'user physiquement de façon à amoindrir ses capacités ultérieures à attaquer et défendre. Le plaquage est explicitement autorisé ${ }^{13}$, avec pour seules restrictions l'interdiction du plaquage haut, ou "cravate», les plaquages par anticipation ou à retardement et ceux effectués sur un joueur ne portant pas le ballon. Dans la mesure où ces considérations valent aussi bien, à quelques détails réglementaires près, pour le rugby à XIII et le football américain que pour le rugby à $\mathrm{XV}$, en quoi peut-on marquer la spécificité de ce dernier au sein des sports de combat collectif? Il nous semble qu'elle réside essentiellement dans les modalités spécifiques qu'y prennent les regroupements de joueurs dans des phases statiques ou dynamiques : le maul ou groupé-pénétrant, la mêlée ouverte et, surtout, la mêlée ordonnée, qui n'ont pas de véritable équivalent dans le rugby à XIII ou le football américain. Nous allons ici nous attacher à montrer que la mêlée est le creuset d'une certaine conception du groupe orientée vers la solidarité.

Afin de bien comprendre de quoi il s'agit, faisons un détour par un certain nombre de considérations techniques. Plus peut-être que les autres phases de jeu en rugby à XV, la mêlée ordonnée est en effet particulièrement opaque au profane, qui n'y voit souvent que mystère et confusion. machines », « cage » ou « mine » (puisqu'on y « va au charbon »)... L'âpre combat qui s'y 
mène exige bien entendu des pratiquants un certain nombre de qualités morales que les intéressés eux-mêmes rangent sous le vocable passe-partout de "vaillance " (où l'on retrouve le courage, la persévérance dans l'effort, la capacité à supporter la souffrance sans fléchir...). Mais il s'exerce également à partir d'un savoir-faire technique dont l'analyse est fondamentale pour notre propos et qui revêt des aspects à la fois individuels et collectifs.

Il n'est sans doute pas exagéré de dire que l'essentiel de ce savoir-faire réside dans la maitrise technique des appuis, qui permet une bonne entrée en mêlée, laquelle conditionne à son tour une poussée efficace. Par appuis, il faut entendre ici ceux qui s'exercent sur la partie haute du corps: le cou, la tête, les épaules et le dos ${ }^{14}$. C'est évidemment dans l'affrontement des deux premières lignes que le problème se pose avec le plus d'acuité.

Le premier souci d'un avant qui entre en mêlée est d'avoir un dos plat. Le contact entre deux piliers adverses (A et B) se fait au niveau de l'épaule et du cou. Pour un pilier gauche A face au pilier droit adverse, la tête étant toujours placée à gauche, l'épaule d'appui sera toujours la droite, l'épaule gauche restant libre. Dans un premier temps, l'épaule droite de A entre en contact avec la base dorsale du cou de B; puis, lorsque la pression augmente, le lieu du contact change. Supposons que A pousse plus fort que B : il lui fera courber le cou, puis le dos. $B$ se retrouvera dans une position très inconfortable et ne pourra pas transmettre efficacement la poussée provenant de ses deuxièmes et troisièmes lignes. Tout l'art d'un pilier consiste donc, lorsqu'il entre en mêlée, à éviter de fléchir le cou, en engageant sa nuque assez loin sous la poitrine du joueur adverse; pour y parvenir, il lui faudra éviter de donner son cou en appui à l'épaule droite de l'adversaire, donc placer son épaule gauche en dessous de cette épaule droite. C'est à ce prix qu'il pourra présenter, à l'entrée en mêlée, un dos droit, gage de l'efficacité de sa poussée et de celle de ses partenaires.

Dans la mesure où il n'y a qu'une bonne position pour deux, c'est évidemment au moment de l'impact entre les deux premières lignes que se joue le bon positionnement. Mais cette phase de jeu essentielle est précédée de quelques secondes par une sorte de "pré-action » qui consiste, avant le signal d'entrée en mêlée donné par l'arbitre, à préparer ses appuis. C'est là que se noue la dimension la plus collective de l'engagement physique, notamment dans la façon dont les deux piliers et le talonneur d'une même équipe se lient les uns aux autres. Plusieurs solutions techniques sont ici possibles. 1) Les deux piliers peuvent ceinturer le talonneur avec le bras intérieur. Très efficace dans la mesure où elle réalise le rapprochement maximum entre les piliers et le talonneur, cette technique risque cependant de mettre ce dernier en danger en cas d'effondrement de la mêlée car il lui est plus difficile de se détacher. 2) Les deux piliers se lient l'un à l'autre directement au niveau des épaules. Bien soudés, ils ne mettent pas en danger le talonneur en cas d'effondrement. 3) Le pilier droit saisit le pilier gauche par l'épaule, ce dernier prenant son partenaire par le haut de la culotte. La cohésion de la première ligne est excellente et le talonneur est assez libre de ses mouvements.

Quant aux deux deuxièmes lignes, ils ont à résoudre deux problèmes principaux de liaison : celle avec le couple pilier/talonneur, et celle avec leur co-équipier de la même ligne. Le deuxième ligne place son épaule en dessous du pli fessier du pilier et, le plus souvent, engage son bras entre les jambes du pilier afin de saisir la ceinture de son short. Il peut ceinturer son partenaire de la deuxième ligne de deux manières : soit en 
passant la main de son bras intérieur sous l'aisselle du partenaire, soit en le saisissant par la ceinture du short.

Des quelques notations techniques qui précèdent, il résulte qu'au-delà d'une nécessaire synergie entre avants, le fait d'être liés physiquement sous l'effort intense -et le fait d'être liés de cette manière particulière, faite d'une grande proximité charnelle-crée une communauté de sensations et d'intérêts extrêmement concrète. Il n'existe sans doute pas de sport collectif où l'on touche l'autre de manière aussi intime. Or -et ceci nous paraît absolument déterminant-, cet autre est à la fois un co-équipier et un adversaire. D'une part, on l'a vu, la position en mêlée d'un joueur occupant le poste de deuxième ligne implique que sa tête soit prise en étau entre les fesses du pilier (droit ou gauche) et celles du talonneur, mais aussi qu'il passe son bras entre les cuisses du pilier pour l'agripper par la ceinture du short, par devant, touchant ainsi ses parties génitales... Situation inimaginable dans n'importe quel autre sport collectif. D'autre part, cette intimité se double d'une autre : celle qui, au moment de la poussée en mêlée, réunit les deux premières lignes adverses, épaule contre épaule, cou contre cou. D'une certaine façon, on pourrait dire que, tout au moins en première ligne, la proximité est plus grande entre adversaires qu'entre co-équipiers -et cela, au plus fort du combat !

Cette configuration particulière, on le répète, est unique dans le domaine sportif ${ }^{15}$. Elle se trouve en outre redoublée, dans le rugby à $\mathrm{XV}$, par une série d'autres dispositifs réglementaires qui concernent certaines phases dynamiques dans le cours du jeu: mauls (regroupement de joueurs autour du ballon qui est porté par un joueur) et mêlées spontanées ou ouvertes (regroupement autour du ballon qui se trouve au sol). Dans le premier cas, la lutte pour progresser en direction du camp adverse (ou pour stopper la progression des adversaires) est éminemment collective, et suppose là encore une extrême proximité des corps dans le combat. Dans le second cas, l'effort pour obtenir le ballon suppose que certains joueurs «fassent le ménage » autour de celui-ci, pour éviter que des adversaires ne viennent s'en emparer ou pour faciliter son extraction par un co-équipier; travail obscur où se manifeste au plus haut point l'idée de sacrifice au profit du groupe.

«La solidarité signifie qu'il y a des individus qui sont prêts à souffrir au nom du groupe et qui attendent des autres le même comportement en leur faveur. Toutes ces questions sont difficiles à examiner sereinement, car elles touchent à notre sentiment intime de la loyauté et du sacré. Quiconque a accepté la confiance de quelqu'un, en a exigé un sacrifice ou a volontairement donné lui-même l'un et l'autre, connaît la force du lien social. » (Douglas 1999 : 25).

Confiance, sacrifice, sentiment intime de la loyauté et du sacré... À voir fonctionner une équipe de rugby, sur le terrain comme, bien souvent, dans la vie quotidienne, on ne peut qu'être frappé par la prégnance de ces valeurs que Mary Douglas assigne à l'exercice effectif de la solidarité au sein d'une communauté, et que nous faisons découler ici, pour le cas particulier du rugby à $\mathrm{XV}$, de ces dispositifs uniques que constituent les différentes formes de regroupement dans le jeu (mêlée ordonnée, mêlée ouverte, maul...).

Excès et transgressions

Mais ces dispositifs ont d'autres conséquences. En premier lieu, les exigences du combat ne sont pas sans influence sur la conception qu'ont les pratiquants de l'esthétique corporelle. Non seulement ils semblent peu valoriser, surtout au niveau des avants, les catégories du beau ou du laid dès qu'il s'agit de leur propre corps (même si, selon l'expression consacrée, il est prévu qu'ils feront tous « de vilains vieux»), mais 
encore, ils ont tendance à instrumentaliser celui-ci dans le registre de la vulgarité : un corps de joueur de rugby est un corps qui « va au charbon ", qui souille son maillot, qui rote, pète et crache, qui se répand en quelque sorte en dehors de lui-même, dégagé de toute censure, prêt à toutes les transgressions sociales. C'est un corps de l'excès. Car il sait d'où il vient, par quels combats il a été forgé, et garde la mémoire des coups, des percussions, des empilements, des torsions de la colonne vertébrale, des crampons adverses et des insultes sous la mêlée.

Mais surtout, il semble que l'on tient là une explication essentielle de l'existence de ces comportements de virilité exacerbée qui singularisent les joueurs de rugby et qui se manifestent de multiples façons en dehors du terrain, qu'il s'agisse des débordements de la troisième mi-temps ou des rapports qu'ils entretiennent avec la gent féminine. Situation qu'Anne Saouter, dans son étude sur le masculin et le féminin dans le monde du rugby, résume par l'expression «jouer à faire l'homme »: afin de ne pas prêter le flanc à des accusations visant d'éventuels comportements homosexuels qui découleraient de cette étonnante proximité des corps ${ }^{16}$, les joueurs ont tendance à « en rajouter » dans le registre des comportements virils et machistes (Saouter 1998 : 122).

L'extraordinaire charge symbolique de la «troisième mi-temps", le succès même de cette expression qui, au-delà de l'importance souvent exagérée qu'on lui attribue, en vient à constituer dans les représentations communes une manifestation essentielle de la spécificité rugbystique, sont là pour rappeler que l'excès (en l'occurrence surtout de boissons alcoolisées) et les comportements transgressifs de l'ordre social qui s'y manifestent souvent, loin de faire référence à un état de nature, s'inscrivent dans une culture.

«L'ivresse elle-même avec toute sa démesure, engendre des comportements qui varient d'un groupe à l'autre, qui prennent sens dans une culture. Si l'on peut admettre que le sujet ivre "ne s'appartient plus", c'est qu'il exprime non pas le surgissement instinctif d'un barbare état de nature, mais, tout au contraire, le code commun, les gestes et les mots prévisibles d'une ivresse qui est toute symbolique. Par là, l'anthropologue ne perd pas de vue la place cérémonielle qu'occupe le partage d'alcool dans bien des sociétés : loin de signifier la déviance, il réinstaure le lien social. » (Fabre-Vassas 1989 : 7).

39 En effet, pour comprendre ce qui est à l'œuvre dans cette ritualisation de la consommation excessive d'alcool, il faut considérer celle-ci non comme un trait pathologique, redevable d'une catégorisation médicale ${ }^{17}$, mais comme un trait culturel s'inscrivant dans un mode de vie.

Dans le petit monde du rugby, la socialisation du boire prend des formes diverses selon les contextes culturels considérés. Mais elle remplit généralement une double fonction : impératif social auquel il peut être dangereux de ne pas se soumettre ${ }^{18}$, elle est aussi moyen de se situer dans la conformité du groupe d'appartenance. Selon le sens commun, la consommation excessive d'alcool est porteuse de déstructuration de l'individu ou du groupe qui s'y livre; ici, par la vertu d'une réaffirmation des valeurs partagées et au moyen d'une ritualisation des comportements, elle joue à l'inverse un rôle de renforcement du lien social ou, si l'on préfère, de perpétuation de la logique de groupe.

41 Enfin, boire, c'est communiquer... L'état d'ébriété est souvent considéré par les joueurs comme devant faciliter l'accession à un niveau supérieur de commu-nication. C'est le moment où les joueurs "peuvent se parler ", qu'il s'agisse de rugby ou d'affaires privées et intimes: l'alcool fait tomber les inhibitions, la chaleur de l'ambiance et la 
sensation de se trouver protégé au sein de la "grande famille» poussent aux confidences... Boire avec un adversaire qui, sur le terrain, vous a fait quelques misères, c'est une façon d'intégrer pacifiquement cette étrange cohabitation, très spécifique au rugby à XV, de la solidarité (entre équipiers) et de l'antagonisme (vis-à-vis de l'adversaire) au plus chaud de la mêlée. Comme l'indique avec pertinence Anne Saouter (1998: 192) :

«La situation conflictuelle a véritablement existé pendant le match de par la spécificité du jeu, un sport de contact et de combat. Mais elle ne peut se poursuivre ensuite. Continuer la relation sur ce registre serait oublier la différence entre le jeu sportif et le quotidien, entre le statut de joueur et celui de sujet social vivant dans une société où les règles ne sont pas celles du rugby. Or, l'opposition entre les individus sur le terrain a été si intense, si physique, les joueurs ont tellement été liés l'un à l'autre, tous contre tous, qu'un rappel à la normalité est nécessaire : "on est entre adultes et ce n'est qu'un sport". Se retrouver dans un contexte "rugby", dans un microcosme qui défend un certain nombre de valeurs morales comme la solidarité et la fraternité, par le moyen festif qui est une façon plutôt agréable de revenir à la norme, permet de résoudre une contradiction majeure entre "soi", entre gens du rugby: celui à qui je m'opposais violemment tout à l'heure est un allié partageant la même passion que moi. »

D'une façon générale, la troisième mi-temps tout entière permet de résoudre cette contradiction fondamentale du rugby : bien qu'il ne s'agisse que d'un sport (et que d'un jeu), on s'y fait volontairement mal...

Un test empirique : le rugby à XIII

Les notations qui précèdent ont conduit à mettre en exergue deux dimensions propres du rugby à XV: une conception du collectif orientée vers la solidarité entre ses membres et une forte propension aux comportements valorisant certaines formes d'excès. Nous avons montré que ces caractéristiques découlaient directement des certaines propriétés formelles du rugby, qui prennent leur origine dans les règles $d u$ jeu. La comparaison avec le rugby à XIII -sport collectif de ballon et de combat dont les règles sont à la fois proches et distinctes de celles du rugby à XV- va nous fournir un test empirique de ces hypothèses. De ce point de vue, une comparaison entre le rugby à $\mathrm{XV}$ et le football, par exemple, aurait été moins significative, la dimension de combat faisant défaut au football. Nous pouvons ainsi faire jouer le lien règles du jeu/culture en tentant d'apprécier, dans un premier temps, quelles sont les différences fondamentales entre les deux codes, et dans un deuxième temps comment ces différences peuvent être reliées à des oppositions culturelles fortes.

Peu de choses semblent a priori distinguer le rugby à XV du rugby à XIII : un terrain de configuration et de dimensions identiques, des poteaux semblables, le même ballon ovale, un dispositif général de sport de combat collectif (plaquages, ligne de hors-jeu et nécessité de passer le ballon en arrière créant une ligne de front)... La scission intervenue à la fin du siècle dernier au sein du football-rugby n'est pas comparable à celle qui a vu, quelques décennies auparavant, le football-rugby et le footballassociation prendre des voies radicalement différentes. Néanmoins, si l'esprit général du jeu paraît avoir été préservé, les différences réglementaires ne manquent pas. La plus évidente est bien entendu celle qui fixe le nombre de joueurs. C'est cependant loin d'être la plus importante : ce qui distingue le plus le XIII du XV, c'est certainement la règle du tenu et la disparition de la poussée en mêlée.

On dit qu'un joueur est « tenu » lorsqu'il ne peut plus ni progresser, ni transmettre son ballon. En général, cette situation se produit lorsqu'un joueur en possession du ballon 
est plaqué par un ou plusieurs adversaires et qu'il est maintenu au sol. Mais ce peut aussi être le cas lorsque le joueur est immobilisé debout et dans l'incapacité de passer le ballon à un partenaire. À XV, il faut impérativement lâcher le ballon; s'ensuit alors, soit une phase statique où les avants des deux équipes se disputent la reconquête du ballon, soit la perte immédiate de celui-ci, qui est joué par l'adversaire, au pied ou à la main. À XIII, le joueur plaqué garde le ballon et joue, avec un partenaire et au moins un adversaire, une phase de jeu que la tradition a dénommé $~ «$ tenu $»^{19}$, qui peut se répéter cinq fois de suite sans que le ballon change de camp malgré le plaquage.

Tout le rugby à XIII, avec ses qualités et ses défauts, est inscrit dans cette règle du tenu. En effet, alors qu'à XV, le joueur ne prendra pas le risque, en allant seul au combat, de lâcher le ballon et de donner à l'adversaire la possibilité d'une contre-attaque, à XIII, la quasi-certitude de conserver le ballon autorise à tenter seul sa chance en attaque, alors même que le risque de se faire plaquer est important. Alors qu'à XV, le plaquage d'un joueur donne lieu à un regroupement de plusieurs joueurs qui s'affrontent pour le gain ou la conservation du ballon (avec des phases de jeu collectives pendant lesquelles des joueurs peuvent "faire le ménage » autour des regroupements sans pour autant se disputer le ballon lui-même), à XIII, seul le couple plaqueur/plaqué est directement mobilisé dans l'action, les autres joueurs pouvant se déployer en défense et en attaque. D'une façon générale, les phases de jeu centrées sur le combat collectif (mêlées spontanées et groupés-pénétrants) disparaissent à XIII au profit d'actions plus individuelles.

De plus, les mêlées fermées ne servent plus de point de départ à de vastes offensives des lignes arrière, car elles sont de plus en plus négligées et ne donnent lieu à aucune véritable poussée. C'est toute la dimension du combat collectif cristallisé au sein du dispositif unique de la mêlée qui, là encore, disparaît à XIII.

Cela renvoie finalement à la différence qui nous paraît déterminante, à savoir le clivage entre comportements solidaires et comportements individualistes, dont l'origine réside pour l'essentiel dans les deux façons opposées de considérer ces phases de jeu fondamentales que sont le tenu et la mêlée. Ces deux dispositifs aboutissent en effet à deux logiques internes distinctes. Nous avons abondamment illustré celle qui caractérise le XV; pour mettre en scène celle du XIII, nous confronterons nos observations de terrain avec les analyses de Robert Fassolette, Directeur Technique National du rugby à XIII en France et auteur d'un mémoire pour le Diplôme d'Études Supérieures de l'INSEP sur l'histoire des relations conflictuelles entre les deux sports (Fassolette 1996).

En dépit d'un ton souvent très polémique qui renvoie manifestement à l'histoire de ces conflits récurrents, R. Fassolette oppose de façon judicieuse les deux logiques internes. Pour lui, les phases d'affrontement entre avants constituent une "globalité fusionnante [où] le joueur impliqué devient un simple exécutant obéissant mécaniquement aux directives qu'il reçoit, car il ne peut avoir une vision d'ensemble du jeu » [...]; il ajoute :

«Le plaqué est donc temporairement exclu du jeu en tant que joueur et il ne devra son salut qu'au prompt sacrifice collectif de ses partenaires venant à son secours, lui permettant ainsi d'éviter le châtiment que ne manquerait pas de lui infliger l'adversaire [...]. Sans ses partenaires, le joueur plaqué est potentiellement en danger et seul l'esprit de corps ${ }^{20}$ de ses camarades va lui permettre de reprendre part au jeu : sans ce groupe, il n'existerait plus. En fait, on attend de lui exactement la même attitude si un autre joueur se trouve également en situation difficile. Sur le terrain comme dans la vie de tous les jours, symboliquement mise en scène pendant le match, la survie de l'individu face à l'adversité sportive (ou sociale) et le maintien 
de son appartenance à l'équipe (ou à la classe sociale) dominante sont donc dépendants de son entière et inconditionnelle adhésion aux normes et aux règles de fonctionnement, édictées par son groupe sociétal [sic], qui font qu'il doit impérativement et en priorité se mettre au service de ce groupe s'il veut avoir une chance de maintenir son existence en tant qu'individu dominant. »

50 À l'inverse, «le tenu treiziste marque la prise en compte de l'individu-joueur dans l'action en cours, de son respect et de sa responsabilisation en tant qu'acteur du système de jeu... ». En effet, autorisé à tenter seul sa chance en attaque, n'ayant pas besoin de former des regroupements et donc pouvant se passer du soutien de ses coéquipiers, le joueur treiziste valorise un mode d'action fondé sur la seule responsabilité individuelle.

51 A contrario (et pour s'en moquer), Robert Fassolette cite l'opinion de Garrick Morgan, "grand joueur de rugby à XV australien, élu meilleur joueur mondial de la saison internationale 1993, passé à XIII puis revenu dans le giron du XV » :

«Le XV est mon sport mais il m'a fallu transiter par le XIII pour le comprendre. Une chance que les circonstances [le passage du XV au professionnalisme en 1995] m'aient permis de revenir aussi vite [...]. C'est peut-être l'esprit du XV qui m'a le plus manqué [...]. Le XIII est avant tout un jeu individuel, un sport d'égoïstes. [...] Il vaut mieux, une fois sur le terrain, oublier le reste de tes partenaires. [...] la structure est telle qu'il est toujours facile de désigner une brebis galeuse en cas d'échec. Aucun risque à XV, même avec l'arrivée des dollars, le rugby restera un sport fondamentalement collectif. » (cité in Fassolette 1996).

52 Par ailleurs, le cas de la mêlée ordonnée à XV, dont nous avons montré plus haut qu'elle était, pour reprendre les termes de P. Conquet (1994), un «formidable creuset de solidarité ", n'est en rien transposable à XIII, non parce qu'elle n'existe pas, mais parce qu'elle ne donne lieu à aucune poussée, et donc à aucun combat collectif. C'est aussi à l'absence de ce dispositif structurant qu'il faut attribuer, dans le contexte treiziste, la valorisation de l'individu, le défaut relatif de convivialité, la non-existence de modes de régulation collective de la solidarité comme la troisième mi-temps et l'absence de référence à la « grande famille du rugby »...

Variations dans le temps et dans l'espace

53 En toute rigueur, les développements qui précèdent doivent aboutir à la conclusion suivante : s'il est vrai que les règles du jeu du rugby à XV, qui traduisent une logique spécifique, ont donné naissance à des propriétés formelles aboutissant, par le façonnage des corps et des esprits, à une culture également spécifique, il est clair que cette culture doit être présente chez tous les pratiquants du rugby à XV en quelque point de la planète où ils se trouvent, dans la mesure où les mêmes règles du jeu doivent s'appliquer à tous. Or, bien entendu, la réalité est beaucoup plus complexe...

54 Tout d'abord, il faut prendre garde à ne pas figer la culture du rugby dans une sorte d'atemporalité : à l'image de toute autre configuration culturelle, celle que nous étudions est éminemment sensible aux influences du temps qui passe. On se bornera ici à souligner deux ordres de fait.

55 En premier lieu, les règles du jeu elles-mêmes ne sont pas immuables, et celles du rugby à XV moins encore que bien d'autres : depuis 1948, elles ont été modifiées à 18 reprises, et certaines modifications ayant concerné plusieurs règles à la fois, c'est au total plus de 50 règles qui ont changé pendant cette période, soit environ une par an en moyenne... Toute la question est ici de savoir si ces évolutions sont suffisamment importantes pour toucher aux « fondamentaux » du jeu et, par conséquent, influencer 
la combinaison des traits culturels correspondants. Pour l'immense majorité des modifications réglementaires, la réponse est probablement négative; mais que l'on pense simplement à la règle édictée en France il y a quelques années pour les séries inférieures, qui interdit la poussée en mêlée ordonnée afin d'éviter, selon ses promoteurs, les accidents graves en cas d'effondrement: c'est l'un des fondements essentiels de la culture rugbystique qui est ainsi menacée ${ }^{21}$.

En second lieu, l'irruption du professionnalisme dans le rugby à XV, officielle depuis 1995, a bouleversé bien des aspects d'un sport jusque là très marqué par les valeurs de l'amateurisme. Un amateurisme, certes, souvent qualifié de «marron »... L'International Board a violemment condamné le rugby français, dans les années trente, pour non respect de la règle qui veut qu'un joueur amateur ne soit pas rémunérée ${ }^{22}$. De fait, l'argent a fort bien circulé dans le rugby français pendant toute la période où régnait l'amateurisme; simplement, il s'agissait de pratiques relativement discrètes, en tout cas non officielles, et les sommes en jeu étaient beaucoup plus modestes que celles d'aujourd'hui. Les modifications qui résultent de cette nouvelle situation juridique sont loin d'être négligeables. Tout d'abord en termes de statut $d u$ joueur qui, censé pratiquer moyennant rémunération, devient salarié d'un club, soumis à des «conditions de travail» plus draconiennes (en termes d'horaires, d'entraînements quotidiens, de séances de musculation, de règles d'hygiène de vie et de diététique, d'obligations de représentation publique imposées par les divers types de sponsors contribuant à financer le club qui les emploie...). Mais surtout du fait que ce qui est en jeu dans l'imposition d'une logique de professionnalisation, c'est bien évidemment la substitution du pouvoir économique au pouvoir sportif. Pour ne citer qu'un exemple, du côté du Stade Français -le club de rugby français qui, sous l'influence de son président Max Guazzini, vice-président de la radio NRJ, est sans doute allé le plus loin dans cette substitution-, l'affaire est entendue: le "rugby cassoulet » a vécu, laissant la place aux pom-pom girls, à un hymne du club (I will survive) diffusé à des centaines de milliers d'exemplaires, à une petite voiture téléguidée pour amener le tee auprès du joueur qui va botter une pénalité, et à des extraits de la chanson Un, dos, tres, Maria de Ricky Martin chaque fois qu'un tir au but est réussi... La mutation qui est en train de se produire est bien celle de l'irruption, non du spectacle (car le rugby, comme la grande majorité des sports, est d'emblée et nécessairement spectacle), mais de sa médiatisation. Ceux qui tiennent désormais les rênes du pouvoir sont bien des hommes de médias, pour lesquels le rugby constitue un créneau qu'ils jugent rentable ${ }^{23}$.

Il est bien clair que les menaces qui pèsent, d'après de nombreux observateurs, sur les "valeurs du rugby " produisent des « discours de crise » marqués par la nostalgie d'un monde considéré comme irrémédiablement condamné. Parfois même, la disparition progressive du système de valeurs sur lequel repose la culture traditionnelle du rugby suscite chez certains pratiquants des réactions de révolte ( il n'est pas question de manger de ce pain-là »). Mais nul ne s'y trompe cependant : le rugby d'élite est engagé dans une évolution dont on voit mal comment elle pourrait être remise en question, et le sursis dont paraît bénéficier le rugby des niveaux inférieurs, qui ne peut prétendre au professionnalisme, n'est à terme qu'un trompe-l'œil. En effet, non seulement c'est toute l'image d'un sport qui se transforme, mais la nécessité absolue d'une loi commune à tous les niveaux hiérarchiques du jeu, de la 4ème série régionale à la première division -loi commune essentiellement représentée par les règles du jeu-, ne peut que tendre à une uniformisation générale des pratiques, des comportements et des représentations du monde de l'ensemble des pratiquants. Là encore, la question est 
de savoir si ces bouleversements seront suffisamment importants (ou toucheront des points suffisamment sensibles) pour que les «invariants" en soient eux-mêmes menacés.

La seconde nuance qu'il faut apporter au modèle présenté ici réside dans la nécessité de prendre en compte le contexte culturel local dans lequel vient s'insérer la pratique. C'est sur cette dimension contextuelle que nous allons à présent insister, en privilégiant deux entrées différentes.

L'éternelle question de la diffusion géographique

Lorsque l'on cherche à expliquer pourquoi le rugby à XV, à l'inverse du football, n'est absolument pas pratiqué dans un grand nombre de pays -ou, à une autre échelle, pourquoi il s'est davantage concentré dans le sud que dans le nord de la France-, on mobilise généralement un certain nombre de données historiques: les responsables d'un tel état de fait sont alors des institutions, voire des individus ou des groupes, dont l'action a été jugée déterminante dans un sens ou dans l'autre ${ }^{24}$. De telles analyses sont fort utiles pour comprendre comment s'est effectuée la diffusion géographique. Malheureusement, elles ne permettent pas de répondre à la question essentielle, à savoir: pourquoi les choses se sont-elles passées ainsi et non autrement? L'histoire événementielle, on le sait, n'est jamais explicative, elle est précisément ce qu'il faut expliquer. Si, pour reprendre l'exemple français, on cherche à comprendre pourquoi, au début du XIXe siècle, la pratique du rugby s'est progressivement étiolée dans le nord et renforcée dans le midi de la France, ou pourquoi des initiatives comme celles du docteur Tissié et de la Ligue girondine d'éducation physique (Augustin 1989) ont pu naître et prospérer dans cet endroit du monde mais ne pas voir le jour (ou rester lettre morte) ailleurs, il nous semble qu'il est nécessaire de mobiliser des éléments de réflexion plus spécifiquement anthropologiques, qui se situent au croisement des propriétés formelles du rugby et des contextes culturels dans lesquels vient s'insérer la pratique.

60 Afin d'étayer cette proposition 闗qui, on l'aura compris, ne rejette pas la dimension historique, mais tente de l'intégrer au sein d'une approche anthropologique des pratiques sportives睅, il est clair qu'un travail d'enquête considérable doit être entrepris, notamment par la multiplication des monographies comparatives entre contextes culturels différents et entre divers types de sports. Un tel travail n'en est encore qu'à ses débuts. On voudrait cependant donner un aperçu du type de questionnement qu'il implique en prenant l'exemple du sous-continent indien ${ }^{25}$.

61 L'Inde, qui compte environ un milliard d'habitants, n'a guère obtenu sur le plan sportif de véritable notoriété, si l'on excepte le polo, le hockey sur gazon et le cricket, sports du reste relativement peu répandus dans le monde; par ailleurs, l'influence anglaise n'est pas allée jusqu'à y imposer le rugby à XV, quasiment inexistant en cette partie du monde. Peut-on invoquer, ici encore, des raisons purement institutionnelles et historiques? Ne faut-il pas plutôt se tourner du côté des spécificités culturelles pour expliquer ces particularités de la diffusion du rugby? On pense notamment au régime des castes, aux cloisonnements et aux interdits qu'il suscite au sein de la population, et qui pose a priori deux types de problèmes. En effet, parmi les trois traits fondamentaux du système des castes - séparation, division du travail, hiérarchie (Bouglé 1993; Dumont 1966), deux posent question dans la perspective de la pratique sportive : la séparation (interdiction des contacts directs ou indirects entre membres de castes différentes, qui régit encore largement, malgré les influences coloniales et l'évolution du monde moderne, les relations sociales) et la hiérarchie, contraire à l'idéal sportif 
d'égalitarisme absolu qui veut que chaque compétiteur soit placé dans les mêmes conditions que les autres (système " démocratique ").

À première vue, l'impératif hiérarchique impliquerait une réticence généralisée des Indiens par rapport à l'ensemble du système sportif -ce qui, nous l'avons vu, n'est pas le cas. Mais sans doute faut-il faire appel ici à l'extrême complexité du contexte culturel indien. Même si l'on peut montrer que la colonisation anglaise et, plus tardivement, l'évolution du monde moderne et des échanges économiques ont pu tempérer le rôle effectif des castes en Inde, il reste d'une part, que les principes qui sont au fondement du système ("l'état d'esprit », pour parler comme Dumont) sont encore très présents à l'heure actuelle ${ }^{26}$, et d'autre part, que le problème de l'implantation des sports en général et du rugby en particulier se posait en Inde dès la fin du XIXe siècle et tout au long de la première moitié du XXe siècle.

Une illustration de cette complexité est fournie par Heinrich \& Landy (1995). Pour expliquer que, malgré l'existence de très nombreuses castes hiérarchisées selon des degrés de pureté relative, on n'observe pas une véritable atomisation de la société ou une simple juxtaposition, les auteurs font appel à la notion de cosmopolitisme (adoption d'un code culturel universel fondé sur certaines valeurs occidentales, surtout anglosaxonnes) qui prend des formes différentes suivant les pays et qui, en Inde, ne chasse pas pour autant les codes traditionnels (multiplicité des références culturelles dont dispose chaque individu) ${ }^{27}$. Cette notion ne doit pas être confondue avec celle d'occidentalisation -la « westernisation », telle que Srinivas $(1952,1966)$ l'a définie-, à savoir « l'interdiction par le colonisateur britannique des sacrifices humains ou de la crémation des veuves, [c'est-à-dire] l'humanisme avec son égalitarisme, l'abolition de l'intouchabilité, aussi bien que la sécularisation des repas dans certaines familles pour qui manger n'est plus un acte religieux.» (Heinrich \& Landy 1995:9). Le cosmopolitisme, lui, ne va pas forcément dans le sens d'une imitation de l'Occident, il conserve une cohérence et une homogénéité propre, il reste plus «indianisé » 㘋c'est par exemple le fait qu'un homme et une femme ne peuvent pas se donner la main en public. Ainsi, d'après les auteurs, « la cosmopolitisation permet la relative permanence de l'indianité face à l'occidentalisation mondiale -mais aussi la persistance du système des castes au détriment d'une véritable démocratie - tout autant que le maintien de la stabilité dans la diversité [...] 》 (Heinrich \& Landy 1995 : 21).

On se trouve donc face à un système particulièrement complexe, segmenté, qui semble d'emblée peu hospitalier à l'idée de transversalité, d'homogénéité et d'universalité que véhicule la notion de sport. Au-delà des problèmes spécifiques que pose une activité comme celle du rugby à XV, c'est donc l'ensemble du système des sports effectivement pratiqués en Inde qu'il faut interroger, en analysant les espaces (sociaux, culturels aussi bien que géographiques) qui les accueillent ou les rejettent.

Il n'en reste pas moins que le rugby à XV revêt des particularités, lesquelles viennent enrichir le tableau et pourraient expliquer sa quasi-absence de diffusion en Inde, ce qui nous renvoie au premier impératif du fonctionnement du système des castes: la séparation. En effet, on l'a vu abondamment dans les développements qui précèdent, les propriétés formelles du rugby font la part belle à une forme de combat collectif fondé sur le contact étroit entre les corps, que ce soit dans les phases de plaquage, dans le dispositif de la touche ou dans les postures qu'imposent les regroupements sous toutes leurs formes (mêlées ordonnées ou spontanées, groupés-pénétrants...). Il est intéressant de constater que les sports les plus prisés par les Indiens sont le cricket, le 
polo, le hockey sur gazon, le tennis, le golf, dont la pratique n'impose pas de promiscuité corporelle comme le handball, le football américain ou, a fortiori le rugby.

Une validation empirique de cette incompatibilité structurelle entre les propriétés formelles du rugby et le contexte culturel indien nous est fournie dans l'archipel des Fidji, où l'essentiel de la population se partage à peu près également entre Mélanésiens et Indiens d'origine ${ }^{28}$. Or, ceux-ci ne jouent pas au rugby (mais au cricket, et surtout au football), alors que pour les Mélanésiens, ce sport est le plus passionnément pratiqué29. Les universaux à l'épreuve du contexte

Les exemples de réappropriation culturelle sont fréquents dans le domaine sportif. Nous avons cité, en début d'article, la solution adoptée par les Fox amériendiens. LéviStrauss mentionne également le fait que les Gahaku-Kama de Nouvelle-Guinée, initiés par des missionnaires, pratiquaient le football d'une façon singulière : le jeu prenait fin non pas à l'issue de la période réglementaire (deux fois quarante-cinq minutes), mais lorsque les deux camps étaient à égalité des parties gagnées. Les affrontements, qui pouvaient ainsi durer plusieurs jours, étaient une occasion ritualisée de renforcer la solidarité du groupe en excluant la dimension compétitive du jeu (il faut un vainqueur et un vaincu) (Read, cité par Lévi-Strauss 1962:44). De manière tout aussi radicale, l'importation du cricket aux Îles Trobriand s'est traduite par une réappropriation culturelle conduisant à intégrer étroitement la compétition à certains rituels festifs, mais aussi à modifier en profondeur les règles du jeu (nombre de membres de chaque équipe, rôle dévolu à chacun, etc. $)^{30}$. On voit bien que, dans ces divers cas, l'influence du contexte local a conduit à l'invention d'un autre jeu, répondant à d'autres règles. Bien évidemment, ni les Fox, ni les Gahaku-Kama, ni les Trobriandais n'ont jamais prétendu participer aux compétitions internationales de football et de cricket, car il s'agit moins là d'une interprétation particulière des règles que du remplacement d'un jeu par un autre.

En revanche, la façon dont le rugby est pratiqué, par exemple, dans certains archipels du Pacifique (Îles Tonga, Fidji ou Samoa) manifeste non pas une invention réglementaire (ce qui permet aux Tongiens, aux Fidjiens et aux Samoans de participer aux rencontres internationales, comme la Coupe du Monde), mais une intégration étroite à certains enjeux des sociétés d'accueil, notamment sur les plans politique et religieux. De même, et en analysant des exemples moins exotiques, on peut déceler des façons spécifiques non seulement de jouer au rugby, mais aussi de le vivre ${ }^{31}$, en comparant les rugbys anglais, gallois ou français... ou encore, à une échelle plus réduite, le rugby tel qu'il se pratique dans des régions distinctes d'un même pays.

Bien entendu, nous retrouvons le problème méthodologique souligné plus haut, à savoir la cruelle absence de travaux comparatifs dans ce domaine, qui nous contraint parfois à poser des jalons pour des recherches ultérieures plutôt que de présenter des vérifications des hypothèses avancées. Toutefois, certains éléments de réponse peuvent être apportés dans le cadre français.

70 En comparant Saint-Vincent-de-Tyrosse et Marseille (Darbon 1997a, 1997b), il s'agissait évidemment d'opposer un "rugby des villages » et un « rugby des villes », mais aussi de confronter une localité où la pratique du rugby constitue le fondement essentiel de l'identité locale et une cité où cette pratique, quoique bien réelle, ne bénéficie que d'une visibilité et d'une légitimité extrêmement réduites (« Marseille, ville de foot... »). Mais d'autres lieux que les quartiers nord de Marseille peuvent être mobilisés dans la perspective d'une opposition au "paradigme culturel» représenté par Tyrosse en 
matière rugbystique -notamment Massy en région parisienne (Baudet 1999), ou Le Haut-Vernet dans la banlieue de Perpignan (Roitg 2001). Dans tous ces cas -et sans doute y en a-t-il d'autres, ce qui ne remet pas en cause pour autant leur caractère marginal au sein de la culture rugbystique dominante-, il apparaît que si les "fondamentaux» de cette culture sont bien présents, viennent s'y mêler des spécificités qui lui sont étrangères et qui tiennent au contexte local (type d'habitat, populations concernées, existence d'entraîneurs, également éducateurs militants...); à la logique sportive traditionnelle s'ajoutent, de façon plus ou moins harmonieuse, des logiques très volontaristes d'intégration sociale et de mélange de populations d'origine ethnique différente qui font généralement défaut à l'immense majorité des clubs français.

71 On voit donc où se situe l'intérêt d'une réflexion anthropologique sur les cultures sportives: dans l'analyse des relations complexes entre, d'une part, les dimensions « universelles » ou « invariantes» de ces cultures telles que l'on peut les dériver des propriétés formelles des pratiques correspondantes et, d'autre part, les éléments de variabilité introduits par la prise en compte du temps et des contextes culturels locaux dans lesquels ces pratiques s'insèrent. Partout, il est nécessaire de conforter et d'affiner le modèle par un souci constant de retour au « terrain ».

72 Cette démarche implique en premier lieu que l'on procède à des comparaisons d'une même pratique sportive dans des contextes locaux diversifiés, de façon à tester l'éventuelle invariance des spécificités culturelles qui auront été décrites, ainsi que la variabilité des modalités par lesquelles elles se manifestent.

73 Mais dans la même perspective, il convient en second lieu de procéder à des comparaisons entre sports différents. En prenant l'exemple du rugby à XV et en le comparant avec le rugby à XIII, nous nous sommes limités à deux cas relativement proches (sports collectifs de ballon et de combat), qui par surcroît font partie de la catégorie soumise à la contrainte réglementaire; il est clair que l'analyse, selon des présupposés théoriques analogues, de pratiques répondant à la contrainte naturelle devra induire certains aménagements du modèle. De plus, au sein de chacune de ces catégories, les différents types de pratique devront être distingués, toujours dans la perspective de relier spécificités culturelles et propriétés formelles. Seule la multiplication des travaux comparatifs dans ce domaine permettra de préciser ce qui doit être aménagé et ce qui, pouvant être conservé, en sortira renforcé.

74 Vaste chantier, dont on espère néanmoins qu'il contribuera à montrer qu'il n'est pas moins légitime de réfléchir, en tant qu'anthropologue, sur les pratiques sportives que sur les systèmes de parenté, les mythes ou les pratiques religieuses.

75 Une première version de ce texte a bénéficié de la lecture critique de Marc Bordigoni, Eric Boutroy, Laurence Hérault, Anne Saouter et Frédérice Saumade, que je remercie. Ils ne sauraient être tenus responsables des imperfections du texte définitif. 


\section{BIBLIOGRAPHIE}

Augustin, Jean-Pierre.

1989 « L'étonnante implantation du rugby dans le Midi », Midi, Revue des Sciences Humaines et de Littérature de la France du Sud 4 : 3-22.

Augustin, Jean-Pierre \& Alain Garrigou

1985 Le Rugby démêlé. Essai sur les associations sportives, le pouvoir et les notables. Bordeaux :

Le Mascaret.

Baudet, Marie-Béatrice

1999 « Le ballon contre l'exclusion », pp.166-170, in S.Darbon (ed.), Rugby d'ici. Une manière d'être au monde. Paris : Autrement (« Mutations »).

Bouglé, Célestin

1993 Essais sur le régime des castes. Paris : Presses Universitaires de France (1ère édition 1908).

Bromberger, Christian

1995 Le Match de football. Ethnologie d'une passion partisane à Marseille, Naples et Turin. Paris : Maison des Sciences de l'Homme, (en collaboration avec Alain Hayot et Jean-Marc Mariottini).

1998 Football, la bagatelle la plus sérieuse du monde. Paris : Bayard Éditions.

Conquet, Pierre

1994 Les Fondamentaux du rugby moderne. Aurillac.

Darbon, Sébastien

1995a Rugby mode de vie. Ethnographie d'un club : Saint-Vincent-de-Tyrosse. Paris : Jean-Michel Place («Les Cahiers de Gradhiva »).

1997a Du rugby dans une ville de foot. Le cas singulier du Rugby Club Marseille-ASPTT. Paris :

L'Harmattan.

1997b « La "grande famille" du rugby, entre hospitalité et solidarité », Communications 65.

Darbon, Sébastien (ed.)

1999 Rugby d'ici. Une manière d'être au monde. Paris : Autrement (« Mutations ») nº 183.

Darbon, Sébastien \& Frédéric Saumade

2000 « Le sable et l'argile : à propos des pratiques ludiques et spectaculaires dans les Landes ",

Ethnologie française $3: 445-457$.

Douglas, Mary

1999 Comment pensent les institutions. Paris : La Découverte/MAUSS.

Dumont, Louis

1966 Homo hierarchicus. Le système des castes et ses implications. Paris : Gallimard.

Dunning, Eric \& Kenneth Sheard

1976 « The Bifurcation of Rugby Union and Rugby League ", International Review of Sport Sociology $2: 31-72$.

Fabre-Vassas, Claudine

1989 « La boisson des ethnologues », Terrain $13:$ 5-14 (« Boire »).

Fassolette, Robert

1996 Histoire politique du conflit des deux rugby en France de l'entre-deux-guerres à la guerre 
froide. Origines, conséquences et perspectives analysées au moyen des logiques internes respectives. Mémoire pour le Diplôme d'Études Supérieures de l'INSEP.

Faure, Jean-Michel

1983 « Pour une sociologie du rugby ", Motricité humaine $2: 26-31$.

1987 Sport, cultures et classes sociales. Thèse pour le Doctorat ès-lettres et sciences humaines. Université de Nantes, Département de Sociologie.

Fouquet, Pierre

1951 « Réflexions cliniques et thérapeutiques sur l'alcoolisme », Évolution psychiatrique 11 :

231-251.

Grimble, Arthur

1953 J'ai choisi les îles. Paris : Amiot-Dumont.

Heinrich, Steven Allen \& Frédéric Landy

1995 « Le barbier et ses clients. Usages diversifiés des codes culturels et permanence de l'Inde rurale ", Études rurales $137: 22$.

Lacoste, Yves (ed.)

1993 Dictionnaire de Géopolitique. Paris : Flammarion.

Leach, Jerry W.

1976 « Structure and Message in Trobriand Cricket », Paper presented at the Symposium, Problems of Anthropological Filming, British Association for the Advancement of Science meetings.

Lévi-Strauss, Claude

1962 La Pensée sauvage. Paris : Plon.

Mars, G.,

1987 « Longshore Drinking, Economic Security and Union Politics in Newfoundland », 91-101, in Mary Douglas (éd.), Constructive Drinking. Perspectives on Drink from Anthropology. Cambridge :

Cambridge University Press/Maison des Sciences de l'Homme.

Parlebas, Pierre

1986 Éléments de sociologie du sport. Paris : Presses Universitaires de France.

Pociello, Christian

1995 Les Cultures sportives. Paris : Presses Universitaires de France.

Roitg, Magali

2001 Rugby, éducation, intégration sociale. Ethnographie d'un club, le Foyer Laïque du HautVernet. Mémoire de maîtrise en Ethnologie, Université de Provence Aix-Marseille 1.

Saouter, Anne

1998 Du masculin et du féminin dans le rugby. Jeu des oppositions et gestion des transgressions. Thèse de Doctorat, Toulouse.

2000 « Être rugby " Jeux du masculin et du féminin. Paris : Maison des Sciences de l'Homme.

Srinivas, M. N.

1952 Religion and Society among the Coorgs of South India. Oxford : Clarendon Press. 1966 Social Change in Modern India. Berkeley : University of California Press.

Weiner, Annette B.

1977 Review of : « Trobriand Cricket : An Ingenious Response to Colonialism », American Anthropologist 79 : 506-507.

1978 Review of : «Epistemology and Ethnographic Reality : A Trobriand Island Case Study », American Anthropologist $80: 752-757$. 


\section{NOTES}

1. Tout au moins si on les compare à leurs homologues britanniques ou nordaméricaines.

2. Cf. notamment, dans la lignée des travaux de P. Bourdieu, Pociello (1995), et les intéressantes discussions critiques par Faure $(1983,1987)$.

3. Par pratiquants, on entend ici l'ensemble de ceux qui jouent ou ont joué : ils peuvent donc inclure des dirigeants (de clubs ou d'instances fédérales) ou des spectateurssupporteurs.

4. Comme par exemple comportements collectifs marqués par la solidarité vs comportements individualistes (nous y reviendrons abondamment).

5. Une telle perspective de recherche, on le voit, est radicalement différente de la sociologie préconisée par C. Pociello (1995), qui s'attache à classer les pratiques sportives sur une échelle de hiérarchie sociale.

6. Malgré les efforts des fédérations, lorsqu'elles existent, pour les ramener dans le giron du système sportif « classique »...

7. Ces termes doivent être compris ici comme s'appliquant à des propriétés qui seraient valables dans tous les contextes où s'appliquent les mêmes règles du jeu.

8. Bien entendu, la distinction entre éléments universels et contextuels correspond ici à un artifice de présentation, dans la mesure où l'on n'observe jamais que des manifestations culturelles déjà contextualisées. Mais cet artifice s'impose d'un point de vue analytique comme un passage obligé qui permet d'assigner à la notion de règles du jeu la place qui, selon nous, lui revient.

9. On devrait y ajouter en toute rigueur le football australien et certaines formes de jeux de ballon gaéliques. Mais il s'agit là de sports dont la diffusion ne dépasse pas le cadre des pays qui les ont enfantés.

10. Il s'agit là de jeux interpénétrés et de circulation de balle. Il faut mentionner, toujours dans la même catégorie d'opposition à la balle, le badminton, le tennis (y compris de table) et le volley-ball, qui sont des jeux de renvoi, pour lesquels les deux équipes sont cantonnées dans leur terrain respectif.

11. Certains éducateurs de rugby, soucieux de ne pas effrayer les parents des enfants qu'ils doivent initier à ce sport fondé sur l'affrontement physique, répugnent à parler de « combat » et préfèrent le terme de « contact ».

12. Il s'agit là d'une moyenne à peu près représentative du jeu en vigueur dans le milieu des années quatre-vingt dix. Depuis, elle a eu tendance à augmenter, sous l'influence notamment d'une modification des règles du jeu.

13. Cf. la Règle du Jeu $n^{\circ} 18$, mais aussi la Règle $n^{\circ} 7$ (« Manière de jouer ») qui indique notamment que « tout joueur en jeu peut à tout moment plaquer, pousser ou épauler un adversaire porteur du ballon ».

14. La position des jambes et des pieds n'est pas non plus indifférente, et l'angle formé par la cuisse et la jambe a aussi une incidence sur la puissance de poussée d'un avant, et donc sur la bonne tenue de la mêlée. Mais l'essentiel se joue au niveau de la partie haute du corps.

15. Au rugby à XIII et au football américain, on l'a vu, la mêlée est loin d'avoir la même importance et la même signification. De plus, dans ce dernier sport collectif, le harnachement des joueurs rend moins intimes les contacts éventuels entre joueurs, coéquipiers ou adversaires. 
16. Et sans préjuger, bien entendu, de la réalité de l'homosexualité au sein de ce groupe d'hommes qu'est une équipe de rugby.

17. Un joueur de rugby qui sacrifierait hebdomadairement et sans retenue au rituel de la troisième mi-temps ne serait pas qualifié d' "alcoolique » au sens où le définit la médecine, qui s'en tient assez consensuellement à la définition du Dr Fouquet, pour qui l'alcoolique est celui « qui a perdu la liberté de s'abstenir de boire » (Fouquet 1951).

18. À l'image, dans une autre forme d'activité, de ces dockers canadiens du Newfoundland, qui ne peuvent obtenir du travail que s'ils sont capables de résister à l'ingestion excessive de boissons alcoolisées (Mars 1987).

19. On remarquera que le terme désigne à la fois le fait d'être plaqué et le phase de jeu qui en est la conséquence.

20. Souligné par l'auteur.

21. Bien qu'ayant le statut de test, la mesure a paru suffisamment menaçante pour qu'un grand nombre de piliers se regroupent dans une association dénommée OUGNA (Organisation Universelle des Gros qui N’Avancent), qui mène campagne pour sauvegarder la « culture traditionnelle du rugby ».

22. La condamnation était passablement hypocrite, mais d'autres griefs furent avancés : la « violence » qui régnait sur les terrains, et l'organisation jugée peu orthodoxe du championnat de France. Le contentieux était suffisamment important pour que l'IRB décide, en 1933, l'exclusion de la France du Tournoi des Cinq nations. Jusqu'au conflit mondial, la France dut ainsi se résoudre à rencontrer des nations « mineures » du rugby : Allemagne, Italie, Roumanie...

23. Autre exemple de ce type d'interconnexions au plus haut niveau du rugby français : Pierre-Yves Revol, président du Castres Olympique, est responsable du pôle Communication du groupe Fabre (Laboratoires pharmaceutiques qui soutiennent le club) mais aussi PDG de RMC et de Sud-Radio.

24. Sur le cas français, voir notamment Augustin (1989) et Augustin \& Garrigou (1985). 25. On pourra aussi consulter, concernant la situation spécifique du département des Landes en France, Darbon et Saumade (2000).

26. Dans le rapport entre Brahmanes et Intouchables, par exemple, la réforme gandhiste a beaucoup atténué les interdits, et l'Inde indépendante a déclaré l'intouchabilité illégale; mais cette mesure ne peut transformer du jour au lendemain la situation traditionnelle, concrètement vécue.

27. Par exemple, « un Brahmane, cadre supérieur à Bombay, pourra déjeuner de mouton à la cantine de son entreprise en compagnie d'un collègue de caste inférieure. Mais le soir, une fois rentré chez lui, il consommera une nourriture strictement végétarienne et refusera l'entrée de certaines pièces de son appartement aux personnes de caste inférieure. » (Heinrich \& Landy 1995 : 8).

28. Les Indiens ont été amenés par des compagnies britanniques comme main-d'œuvre sur les plantations de canne à sucre, principale ressource de l'archipel. Alors que les Mélanésiens, et notamment l'aristocratie et les chefs traditionnels, ont une grande influence au sein de l'armée et soutiennent le parti conservateur, les Indiens, particulièrement performants dans certains secteurs d'activité, comptent de nombreux intellectuels et syndicalistes et se regroupent dans un parti travailliste à tendance progressiste. En 1987, les travaillistes remportant les élections, un Premier ministre indien a même été nommé; mais il fut renversé un mois plus tard, au moment où l'équipe nationale de rugby partait pour disputer la première Coupe du monde, par un coup d'État militaire qui restaura le pouvoir mélanésien. À la suite de graves émeutes, 
de nombreux Indiens quittèrent les Fidji, et la Constitution de 1990 entérina la prépondérance politique du groupe mélanésien (Lacoste 1993 : 633). Plus récemment, la communauté indienne ayant à nouveau repris démocratiquement les rênes du pouvoir, un nouveau coup d'État a visé à rétablir un gouvernement mélanésien...

29. Un travail de terrain, que nous avons commencé à mener dans l'archipel des Fidji, s'attache à comprendre les raisons culturelles de ce clivage entre les deux communautés. Ce sera en même temps l'occasion, en analysant les liens spécifiques entre la pratique du rugby et de nombreux aspects de la vie politique et religieuse, de tenter d'apporter un éclairage nouveau -celui des pratiques sportives- sur les travaux anthropologiques déjà réalisés dans l'archipel.

30. Cf. le film Trobriand Cricket: An Ingenious Response to Colonialism, réalisé par l'anthropologue Jerry Leach et le cameraman Gary Kildea en 1974, ainsi que divers commentaires qui en ont été faits (Leach 1976; Weiner 1977, 1978). De même, la manière dont les habitants des Gilbert (à présent Kiribati, Pacifique Sud) jouaient au cricket mettait à rude épreuve le flegme des administrateurs britanniques (Grimble 1953).

31. Le rugby, rappelons-le, est un « mode de vie » autant qu'un sport (Darbon 1995a).

\section{RÉSUMÉS}

L'analyse des cultures sportives qui est proposée dans cet article, à partir du rugby à XV et de sa comparaison avec le rugby à XIII, tente de mettre en évidence les relations entre les propriétés formelles des pratiques sportives considérées (qui se manifestent ici par les règles du jeu) et les combinaisons des pratiques, comportements, rapports au corps et systèmes de valeurs correspondantes. Les « invariants » culturels ainsi définis sont mis à l'épreuve de l'influence des contextes culturels locaux dans lesquels ces pratiques sont immergées, suggérant une nouvelle approche de la question traditionnelle de la diffusion géographique différenciée des sports dans le monde.

For an anthropology of sports. Formal properties and relation to the body in Rugby Union football

Comparing Rugby Union and Rugby League football, the analysis of sports cultures presented in this article, tries to bring out the connections between the formal properties of these games (e.g. the rules) and the combination of the practices, the behaviours, the relations to the body and the values. The invariants thus defined are then examined in the light of their local cultural contexts. Such a method suggests a new approach to the problem of the differentiated geographical diffusion of sports in the world

Por una antropología de las prácticas deportivas. Propiedades formales y relación con el cuerpo en el rugby.

El análisis de las culturas deportivas que se propone en este artículo, a partir de la comparación entre el rugby que se practica con trece o catorce jugadores, intenta poner en evidencia las relaciones entre las propiedades formales de las prácticas deportivas que aquí se consideran (y que se manifiestan a través de las reglas de juego) y las combinaciones de prácticas, comportamientos, relaciones con el cuerpo y sistemas de valores que se corresponden con ellas. 
Así definidos los «invariantes» culturales, se someten a la prueba de la influencia que comportan los contextos culturales locales en los que dichas prácticas se sumergen, sugiriendo así un nuevo enfoque acerca de la cuestión tradicional de la difusión geográfica de los deportes en el mundo.

INDEX

Mots-clés : cultures sportives, propriétés formelles, règles du jeu, rugby

\section{AUTEUR}

\section{SÉBASTIEN DARBON}

CNRS, Institut d'Ethnologie méditerranéenne et comparative (IDEMEC), 5 rue du Château de l'Horloge, 13094, Aix-en-Provence Cedex 2. 